\title{
Propagation path loss characteristics of vertical dipole above perfect ground
}

\author{
Ic-Pyo Hong ${ }^{\text {a) }}$ \\ Department of Information \& Communication Engineering, Kongju National \\ University, 275 Budae-dong, Cheonan, Korea \\ a)iphong@kongju.ac.kr
}

\begin{abstract}
In this letter, we calculated the propagation path loss because of the vertical electric dipole on perfect electric conductor ground in time domain. We derived the electric field in time domain and calculated the transient response and the received signal strength. The results show that the changes in the height of the base station caused the changes in the transient response in time domain and that such changes also affect the changes in received signal strength and the location of the near field because of the effects of ground surface. It is expected that such results can be applied in mobile communication network design and in military communication such as ground surface radars.
\end{abstract}

Keywords: propagation path loss, vertical electric dipole, near field

Classification: Electromagnetic theory

\section{References}

[1] W. C. Y. Lee: Mobile Communication Design Fundamentals (Wiley, New York, 1993) 2nd ed.

[2] M. F. Iskander and Z. Yun: IEEE Trans. Microw. Theory Tech 50 (2002) 662.

[3] S. A. Podosenov, Y. G. Svekis and A. A. Sokolov: IEEE Trans. Electromagn. Compat. 37 (1995) 367.

[4] S. W. Dong, W. Ma, W. Z. Cui, S. Shang, H. Zhang and H. Chen: PIERS Proceedings (2008) 162.

[5] D. Wu and C. Ruan: IEEE Trans. Electromagn. Compat. 41 (1999) 120.

[6] D. L. Sengupta and C. T. Tai: Transient Electromagnetic Fields (Springer Verlag, New York, 1976) 182.

[7] T. K. Sarkar, M. C. Wicks, M. Salazar-Palma and R. Bonneau: Smart Antennas (Wiley, New York, 2003).

[8] S. M. Rao: Time Domain Electromagnetics (Academic Press, London, 1999).

[9] G. S. Smith: An Introduction to Classical Electromagnetic Radiation (Cambridge University Press, New York, 1997). 


\section{Introduction}

Understanding the accurate propagation characteristics is very important for designing wireless communication system with excellent performance [1]. The propagation characteristics generally can be described as propagation path loss, which is large signal characteristics, and fading characteristics, which is small signal characteristic. The information of propagation path loss is very essential for optimizing the coverage and location of the base station in mobile communication. The propagation path loss information can typically be obtained by measuring the electric field intensity. However, this is rather uneconomic and inefficient with regard to time that is needed to extract the data through numerous experiments to be statistically significant. Therefore, it can be understood that accurately and theoretically predicting the propagation path loss is very important.

The propagation path loss models can generally be divided into either experience or theory-based models [2]. In case of experience-based models, the propagation path loss equations can be obtained from statistical test results through electric field intensity measurements such as the Okumura model or the Hata model [1]. In this case, the reliability is very high in similar environments, but parameter modification would be needed to predict the propagation path loss for environments with different frequencies, distances, natural environment and others. Although the theoretical models on propagation path loss have a weakness that ideal conditions are assumed, they also have advantages that the propagation path loss of various propagation environments can be predicted. Because most of the propagation path loss is handled in the frequency domain, there is a shortcoming that the changes in propagation path loss according to the moving speed of the mobile or the transient response received from the mobile is unknown. To overcome such shortcomings, this study predicted the propagation path loss in the time domain, and also applied the radiation analysis of the vertical half wavelength dipole that had been handled in the existing time domain to observe the transient response from the mobile according to the height of the base station. By calculating the radiation characteristics in the time domain that is being radiated from the vertical half wavelength dipole antenna on top of the grounding structure of the perfect electric conductor, the vertical half wavelength dipole antenna was assumed to be the base station, and the radiation characteristics was assumed to be the mobile. Therefore, the transient response of the time domain according to the height of the base station antenna and changes in the propagation path loss characteristics according to the changes in the distance between the base station and the mobile among others were calculated through the time domain analysis between the base station and the mobile.

The results of the study show that the changes in the height of the base station become an important cause for changes in the transient response in time domain and such changes also affect the changes in received signal strength. Furthermore, the observation of the changes in propagation path loss characteristics according to the changes in height of the base station showed that such changes in the height of the base station is an important variable that needs to be considered when designing the mobile commu- 
nication network since it may cause changes in near field location of the base station antenna according to the earth surface.

\section{Time domain electric field of vertical electric dipole}

Fig. 1 shows the structure that was analyzed in this study. The ground is assumed to be the perfect electric conductor and the vertical half wavelength dipole was assumed to be the base station antenna. The total electric field $\vec{E}_{t o t}$ is a sum of the electric field $\vec{E}_{d i r}$, which is radiated because of the dipole on the grounding and the electric field $\vec{E}_{r e f}$, which is radiated because of the image dipole like Eq. (1)

$$
\vec{E}_{t o t}=\vec{E}_{d i r}+\vec{E}_{r e f}
$$

The electric field magnitude in $\theta$ direction that is radiated because of the vertical electric dipole from free space without ground can be shown as Eq. (3) when the vertical electric dipole antenna is a traveling-wave antenna. Consider a transient current $I(t)$ flowing from the feed to the endpoint on a dipole, which is thin and perfectly conducting. If we assume that the time-varying charges are $Q(t)$ at the end point of an antenna, the current distribution is given by D'Almebert's formula as shown below.

$$
I(s, t)=I(t-s / c) \quad s \in[0, L / 2]
$$

Where, $s$ is chosen as parameter of the wire length and $c$ is the velocity of light. Using the current distribution of Eq. (2), the vector potential can be calculated. The magnetic field can be obtained from the vector potential and the electric fields were yielded from the relation $\nabla \times \vec{H}=\partial \vec{D} / \partial t$ as Eq. (3) $[3,5]$.

$$
E_{d i r_{\theta}}=\frac{\eta_{0}}{4 \pi}\left\{\begin{array}{l}
{\left[\frac{2 I\left(t-\frac{r_{1}}{c}\right)}{r_{1} \sin \theta_{1}}-\frac{I\left(t-\frac{L}{c}-\frac{R_{11}}{c}\right)}{R_{11}} \cos \alpha_{11} \cot \left(\frac{\theta_{1}}{2}\right)\right.} \\
\left.-\frac{I\left(t-\frac{L}{c}-\frac{R_{12}}{c}\right)}{R_{12}} \cos \alpha_{12} \tan \left(\frac{\theta_{1}}{2}\right)\right] \\
+c\left[\frac{Q\left(t-\frac{L}{c}-\frac{R_{11}}{c}\right)}{R_{11}^{2}} \sin \alpha_{11}+\frac{Q\left(t-\frac{L}{c}-\frac{R_{12}}{c}\right)}{R_{12}^{2}} \sin \alpha_{12}\right]
\end{array}\right\}
$$

In Eq. (3), $Q(\cdot)$ is a charge distribution function and is given as the integral function of the input current and $\eta_{0}$ is the characteristic impedance in free space. Where, $r_{1}, R_{11}, R_{12}, \alpha_{11}, \alpha_{12}, L$ and $\theta_{1}$ are described in Fig. 1 . In case of existing study results, the assumption of traveling-wave antenna was able to be established because the amplitude of the input current waveform has generally been less than the length of the half wavelength dipole. However in the case of this study, standing-wave antenna that considers the reflection at the end of the dipole must be assumed to obtain the propagation path loss model. Therefore, the electric field magnitude in $\theta$ 


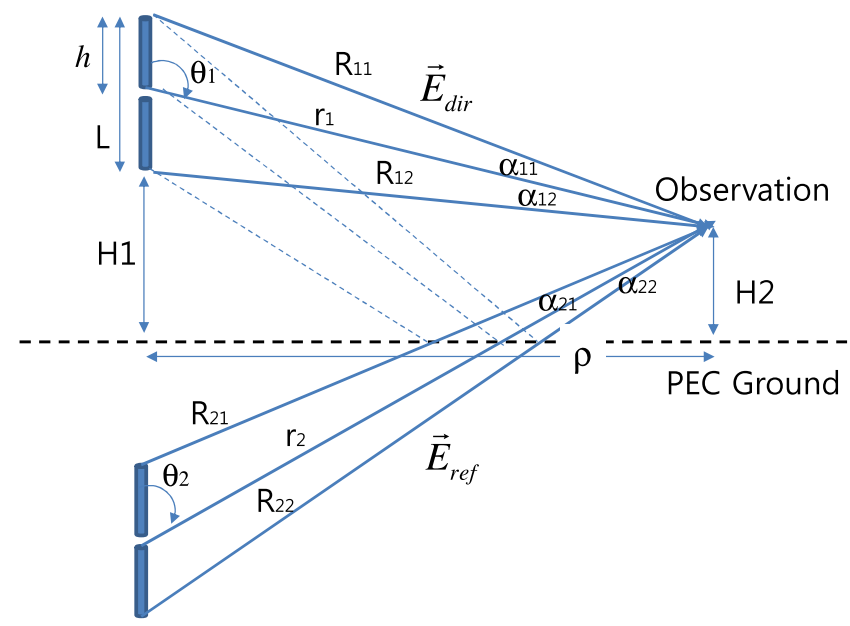

Fig. 1. Vertical electric dipole above perfect electric conductor ground

direction from free space in case of half wavelength dipole can be obtained with Eq. (4) [4]. As pointed out by [4], the standing-wave dipole is equivalent to 4 traveling-wave monopoles. Rothwell's hypothesis is met at the load end of the monopoles that the current is absorbed an the charges accumulate, and the charge-distributions in Eq. (3) can be removed.

$$
\begin{gathered}
E_{d i r_{\theta}}=\frac{\eta_{0}}{2 \pi \rho}\left[\begin{array}{l}
I\left(t-\frac{r_{1}}{c}\right)+I\left(t-\frac{L}{c}-\frac{r_{1}}{c}\right)-I\left(t-\frac{L}{2 c}\left(1+\cos \theta_{1}\right)-\frac{R_{11}}{c}\right) \\
-I\left(t-\frac{L}{2 c}\left(1+\cos \theta_{1}\right)-\frac{R_{12}}{c}\right)
\end{array}\right] \\
E_{t o t_{\theta}}=\frac{\eta_{0}}{2 \pi \rho}\left[\begin{array}{l}
\left.I\left(t-\frac{r_{1}}{c}\right)+I\left(t-\frac{L}{c}-\frac{r_{1}}{c}\right)-I\left(t-\frac{L}{2 c}-\frac{R_{11}}{c}\right) \cos \alpha_{11}\right] \\
-I\left(t-\frac{L}{2 c}-\frac{R_{12}}{c}\right) \cos \alpha_{12}
\end{array}\right] \\
+\frac{\eta_{0}}{2 \pi \rho}\left[\begin{array}{l}
\left.I\left(t-\frac{r_{2}}{c}\right)+I\left(t-\frac{L}{c}-\frac{r_{2}}{c}\right)-I\left(t-\frac{L}{2 c}-\frac{R_{21}}{c}\right) \cos \alpha_{21}\right] \\
-I\left(t-\frac{L}{2 c}-\frac{R_{22}}{c}\right) \cos \alpha_{22}
\end{array}\right]
\end{gathered}
$$

Where, $\rho$ is the distance between base station and mobile and $r_{2}, R_{21}$ and $R_{22}$ are described in Fig. 1. If Eq. (4) considers the grounding surface, Eq. (5) shown below can be expressed by using the parameters in Fig. 1. As previously explained, when the electric field radiated by the half wavelength dipole that has standing wave ratio as obtained from Eq. (5) considers the reflection generated from the signal input end, Eq. (6) below can be derived. In dipole antenna, a portion of each wave of charge, $-R$ stops at the source, and the remainder passes onto the adjacent conductor. The fraction $R$ of each wave of charge is reflected at the source, and no wave of charge passes onto the adjacent conductor. If we repeat this procedure, we can be derived the new amplitudes multiplied by $R$ and the times delayed by $L / c[8]$. Here, $R$ stands for the reflection coefficient from the input end, and $n$ stands for the number of reflection. In this study, parameters of $R=-0.5$ and $n=2$ were used because most electric field are vanished after several reflections. 
To verify the Eq. (6), we calculated the current distribution $I(t)$ on a dipole antenna at $z=0.3 h$ and $z=0.6 h$, respectively, where $h$ is the length between the center and the one end of dipole as describes in Fig. 1. In this letter, Fig. 2 (a) and (b) shows the current distribution plot obtained from Eq. (6) and derived from the method of moments (MOM) in time domain [8]. The results show that the shapes of current distribution are agree well except higher mode current distributions derived from MOM in time domain.

$$
\begin{gathered}
E_{t_{\text {tot }}}=\frac{\eta_{0}}{2 \pi \rho} \sum_{n=0}^{\infty}(R)^{n}\left[\begin{array}{l}
I\left(t-\frac{r_{1}}{c}-\frac{n L}{c}\right)+I\left(t-\frac{L}{c}-\frac{r_{1}}{c}-\frac{n L}{c}\right) \\
-I\left(t-\frac{r_{1}}{c}-\frac{L}{2 c}\left(1-\cos \theta_{1}\right)-\frac{n L}{c}\right) \\
-I\left(t-\frac{r_{1}}{c}-\frac{L}{2 c}\left(1+\cos \theta_{1}\right)-\frac{n L}{c}\right)
\end{array}\right] \\
+\frac{\eta_{0}}{2 \pi \rho} \sum_{n=0}^{\infty}(R)^{n}\left[\begin{array}{l}
I\left(t-\frac{r_{2}}{c}-\frac{n L}{c}\right)+I\left(t-\frac{L}{c}-\frac{r_{2}}{c}-\frac{n L}{c}\right) \\
-I\left(t-\frac{r_{2}}{c}-\frac{L}{2 c}\left(1-\cos \theta_{2}\right)-\frac{n L}{c}\right) \\
-I\left(t-\frac{r_{2}}{c}-\frac{L}{2 c}\left(1+\cos \theta_{2}\right)-\frac{n L}{c}\right)
\end{array}\right]
\end{gathered}
$$

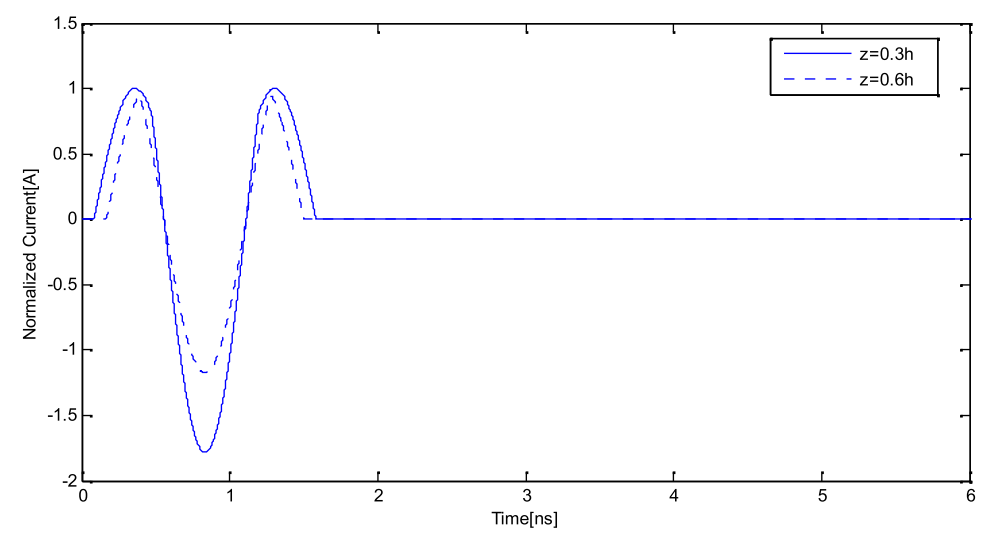

(a) Current distributions on dipole antenna obtained by this letter

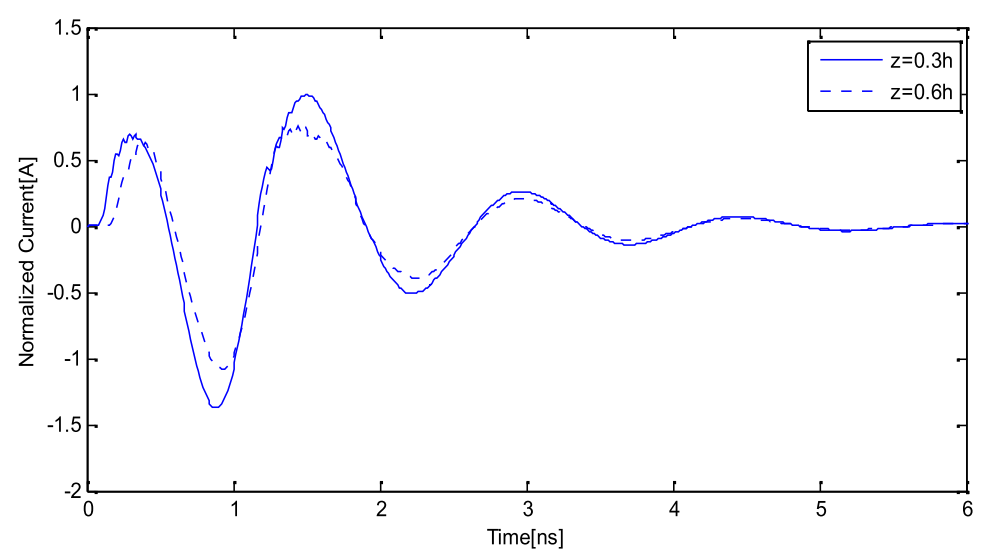

(b) Current distributions on dipole antenna obtained by MOM in time domain

Fig. 2. Comparisons of current distribution between this letter and MOM in time domain 


\section{Simulation results}

We simulated the transient response in time domain by using Eq. (6) derived in the previous section. Transmit frequency of $1.8 \mathrm{GHz}$, height from the ground surface of half wavelength vertical dipole that is assumed to be the base station being $20 \mathrm{~m}$, height of observation that is assumed to be the mobile being $1.6 \mathrm{~m}$, and the distance between the base station and mobile being $100 \mathrm{~m}$ were assumed. All simulations are assumed under the condition of sinusoidal current distribution, i.e., $I(t)=A \sin (\omega t)$ where $A=1$ is the amplitude of the current and $\omega$ is the angular frequency of the signal. Fig. 3 shows the changes in size of $E_{\theta}$ at receiving point when $t=1[\mu s]$, which is after the state of transient response after transmitting sinusoidal wave while varying the height of the base station antenna among the set values in Fig. 3. It can be observed that the size of the received signal strength changes according to the changes in the height of the base station. It can be confirmed that the changes in the height of the base station greatly influence the received signal strength.

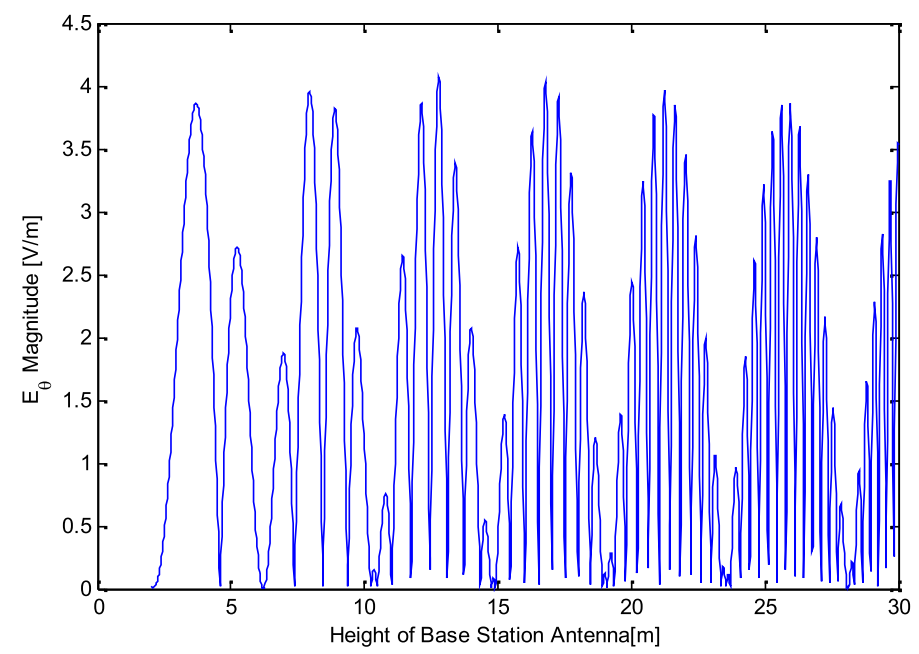

Fig. 3. Changes in received signal strength according to the changes in base station height

Fig. 4 shows the changes on the propagation path loss according to distance when the height of base station is $\mathrm{H} 1=5 \mathrm{~m}, 10 \mathrm{~m}$ and $20 \mathrm{~m}$. From Eq. (6), the mobile tation height $\mathrm{H} 2=1.6 \mathrm{~m}$, the operating frequency is $1.8 \mathrm{GHz}$ and the length of dipole is assumed to be a half-wavelength. From the propagation path loss shown in Fig. 4, the location of appearance of farfield, which is where the received signal stabilizes, is determined according to the height of the base station antenna. The deep fading effect can be observed to decrease at approximate distances of $1.12,4.68$, and $18.8 \mathrm{~km}$ when the height of the base station is 5,10 , and $20 \mathrm{~m}$ as shown in Fig. 4 with arrows, respectively. Such effect is caused by the expansion of the distance of the near field because of the effect of increasing antenna dimension being radiated, including the zero phase antenna of the vertical dipole antenna located on the grounding because of assumption of a perfect electric conductor. When antenna dimension is expressed as $D$ and the wavelength is given as $\lambda$, the near field can be obtained with Eq. (7) as shown below [9]. This can be expressed as Eq. (8) for near field when 


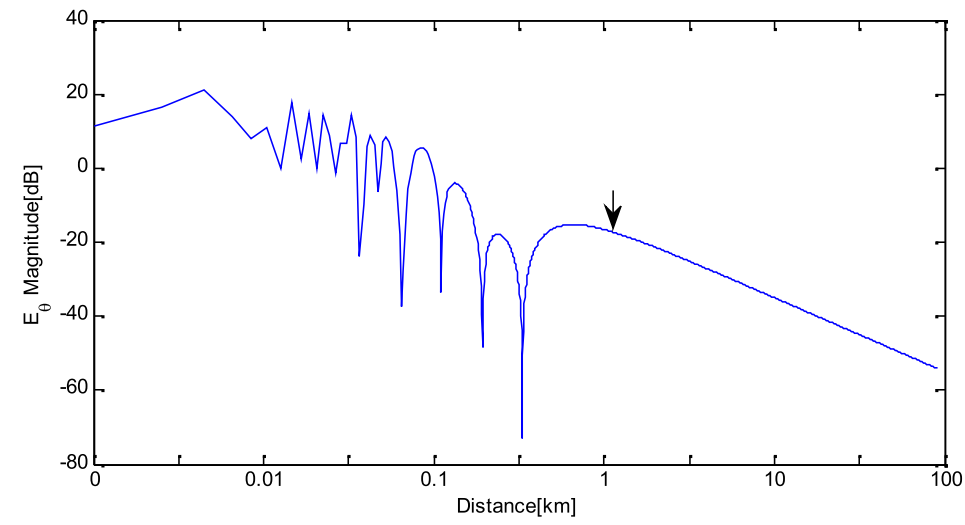

(a) $\mathrm{H}=5[\mathrm{~m}]$

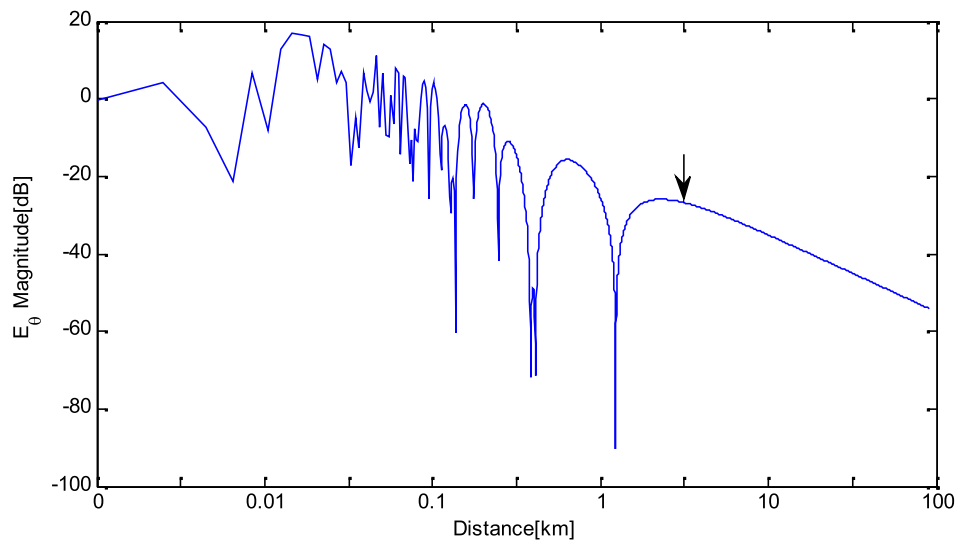

(b) $\mathrm{H}=10[\mathrm{~m}]$

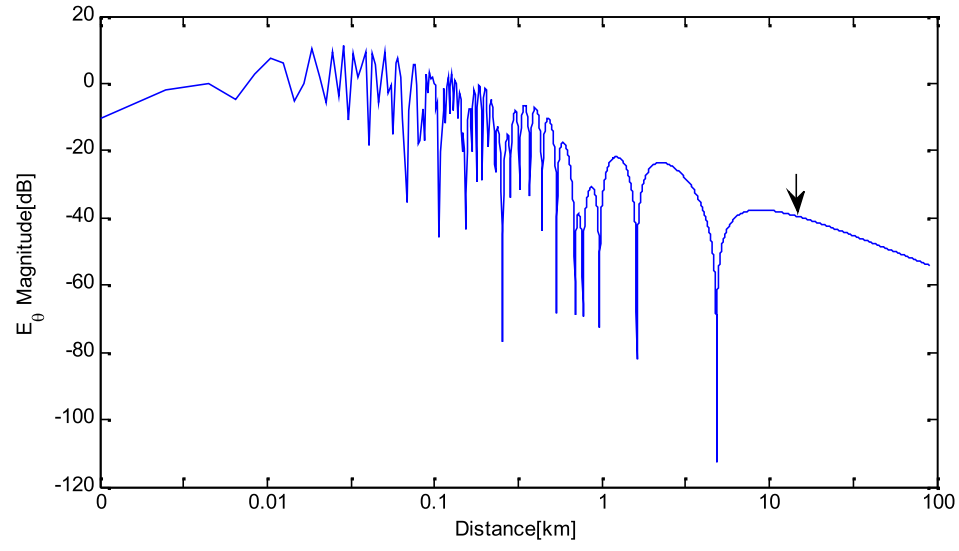

(c) $\mathrm{H}=20[\mathrm{~m}]$

Fig. 4. Propagation path loss for the different heights of base station antenna

modified for application of dipole on perfect electric conductor ground surface as used in this study. From Eq. (7), the $d_{n f}^{\prime}$ for height of the base station $\mathrm{H} 1=5 \mathrm{~m}, 10 \mathrm{~m}$, and $20 \mathrm{~m}$ is $1.2,4.8$, and $19.2 \mathrm{~km}$, respectively, and these results relatively well correspond to the study results from the simulation.

$$
d_{n f}=\frac{2 D^{2}}{\lambda}
$$


Table I. Comparison the far field starting location for different base station height

\begin{tabular}{l|l|l|l}
\hline Base station height[m] & 5 & 10 & 20 \\
\hline Theory[km] & 1.20 & 4.80 & 19.2 \\
\hline Simulation[km] & 1.12 & 4.68 & 18.8 \\
\hline \multicolumn{1}{l}{$d_{n f}^{\prime}=\frac{8 D^{2}}{\lambda}$} &
\end{tabular}

\section{Conclusion}

In this study, the propagation path loss due to the vertical electric dipole on perfect electric conductor ground in time domain was calculated. Half wavelength antenna with standing wave ratio was assumed for the vertical electric dipole and was assumed to be the base station antenna and the mobile was assumed to be located at the observation when calculating the propagation path loss according to the distance between the base station and the mobile and calculating the transient response of time domain according to the height of the base station antenna. With the calculated results, it was confirmed that the height of the base station can alter the received signal strength and the location of the near field because of the effects of ground surface. It is expected that such results can be applied in mobile communication network design and in military communication such as ground surface radars.

\section{Acknowledgments}

This research was supported by Basic Science Research Program through the National Research Foundation of Korea (NRF) funded by the Ministry of Education (2010-0023742) 